\title{
Legal Compliance in Commercial Service Provisioning Across Administrative Domains
}

\author{
Martin Waldburger ${ }^{1}$ and Burkhard Stiller ${ }^{1,2}$ \\ ${ }^{1}$ University of Zürich, Department of Informatics (IFI), CH-8050 Zürich \\ ${ }^{2}$ ETH Zürich, Computer Engineering and Networks Lab (TIK), CH-8092 Zürich \\ \{waldburger, stiller\}@ifi.uzh.ch
}

\begin{abstract}
Internet design principles do not focus on commercial service provisioning. Hence, support mechanisms need to be implemented in order to ensure that value added services can be offered in a competitive context. Commercial product offerings base on contractual agreements concluded between service providers and service customers. Contracts need to reflect business-driven requirements originating from involved contract parties, while they are invariably required to respect those regulations imposed by commerce law. Legal compliance, thus, determines the available range of applicable contractual termsirrespective of whether such a contract governs commercial value added services in the Internet or not. Legal determinations are valid in a limited geographical area. The Internet, however, lacks a distinct notion of location. Consequently, technical means to overcome this fundamental design gap are investigated, in order to ensure that legally compliant contracts can be concluded.
\end{abstract}

\section{Introduction and Motivation}

In order to offer commercial electronic value added services in the Internet respective support mechanisms have to be in place. The minimum range of required mechanisms is determined by those requirements put in order by commerce law. Even though these legislations have a long tradition of being part in civil code determinations, they originally do not consider specifics of electronic service provisioning. Hence, potential adaptations with regard to an application to electronic service provisioning need to be included. Such adaptations comprise mechanisms for Authentication, Authorization, Accounting, Auditing, and Charging (A4C) [8]6] as these elements document the timely course of actions in electronic business transactions.

In the Internet, which is organized as a network of networks, complexity in commercial electronic service provisioning appears to be increased, since trade partners are decoupled from shared room and time experience. In contrast to certain non-electronic business transactions, where physical presence of trade partners might be a sufficient replacement for procedures that explicitly ensure partner identification (authentication) and that assess their respective entitlements (authorization), authentication and authorization are typically mandatory elements in electronic business transactions.

$\mathrm{A} 4 \mathrm{C}$ reflects those functional elements typically required for commercial electronic transactions to be compliant with external or self-imposed regulations. This considers 
the direct understanding of legal compliance. However, legislations traditionally are unaware of electronic commerce. Driven by technological dependencies originating from the TCP/IP-based Internet protocol stack and the Internet's organizational composition from various administrative domains, both, the commercial transaction as such and A4C support mechanisms need to be legally compliant, thus, resulting in an integrated viewpoint on legal compliance.

In this context, different challenging issues arise, out of which the lacking notion of territoriality in the Internet reveals a design gap so fundamental in nature that it potentially menaces legal compliance of commercial electronic service provisioning per se. Commercial value added services base on contracts that are concluded between service providers and service consumers. Contracts are subject to commerce law regulations, whereas these regulations are valid in a geographically limited area, typically within a territory. The Internet, however, lacks a notion of geographical location. Thus, technical means for automated contract conclusion in the Internet have to be investigated, designed, implemented, and evaluated. These mechanisms require legal procedures for international contract formation to be followed and the Internet to be equipped by a notion of location.

Accordingly, the remainder of this paper is structured as follows: Section 2 provides the key legal and technical background relevant for this work. Legal aspects of interest include careful inspection of conflicting law systems and the process of forming contracts in an international context, thus, in a situation where service provider and service customer potentially are located in another legal domain, i.e. another territory. Technical aspects of interest embrace the multi-domain, but extra-territorial nature of the Internet. Based on this background information, the research problem to be addressed is determined in Section 3. whereas the initial approach to a solution and the current status of work are presented in Section 4.

\section{Legal and Technical Background}

For a complete understanding of those consequences implied by territorial nature of (western) law and by the extra-territorial constitution of the Internet, an in-depth investigation into the respective domains of law systems and the organization of the Internet is required. Hence, this section addresses fundamental background information on legal and Internet design principles for contracts that need to be concluded in a global context. In particular, differing notions of domains for regulations (territories) and in the Internet (administrative domains) are outlined. These insights given provide the key arguments to state the research problem to be solved ( $c f$. Section 3 ).

\subsection{Contract Formation Process in an International Context}

Contracts constitute the central element in legal compliance considerations with regard to commercial value added service provisioning. Since the Internet is a global infrastructure, contractual agreements potentially have to be concluded across territorial borders. Law systems of different countries are not fully compatible ( $c f$. Section2.2) so that contractual parties might be required to choose the respective applicable legal system or that arbitration becomes necessary. 


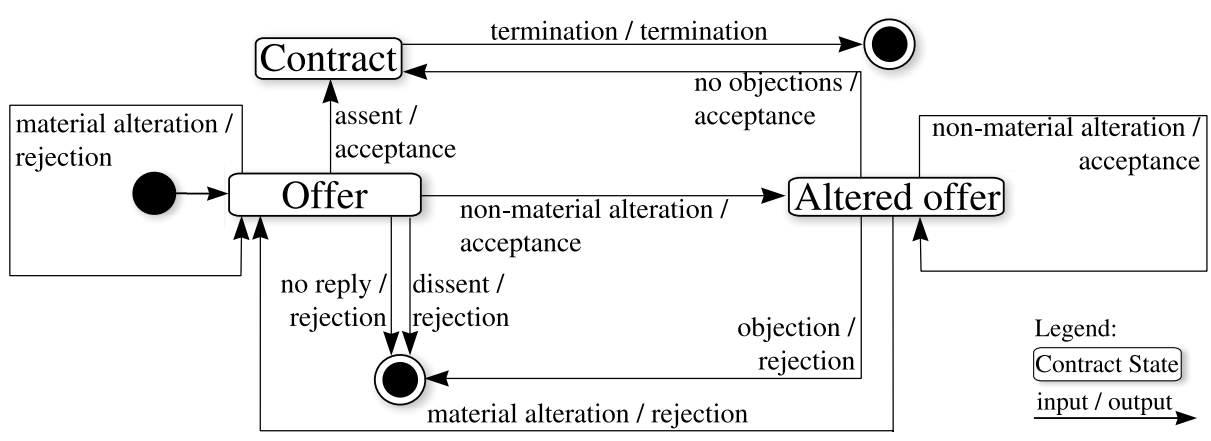

Fig. 1. Contract Formation Finite State Machine Compliant with the United Nations Convention on Contracts for the International Sale of Goods (CISG, [11])

Figure 1 models the process of contract formation for agreements that are governed by the United Nations convention on Contracts for the International Sale of Goods (CISG, [11]). CISG is the most prominent example in law for international contract formation. Depending on the actual situation, other legal determinations need to be considered, e.g., bilateral agreements governing commerce law between two nations.

The depicted automaton visualizes different available possibilities for the so-called offerer (sender of a proposed agreement, called offer) and offeree (the receiver of an offer, an altered or a counter-offer, respectively) in order to consent or dissent on a contract. It includes details on forming a contract, whereas it abstracts from the applicable specifics of contract termination. Figure 1 includes two distinct end states, one for a terminated contract and one for a contract wich cannot be concluded. This separation is important for legal reasons as a terminated contract might imply liability claims.

Upon receipt of an offer, the offeree has two basic choices available. The offeree can either assent or dissent. Assent results in acceptance of the offer, whereas dissent results in rejection of the offer. Assent renders an offer into a contract. Assent subsumes the cases where the offeree assents fully without any modifications to the offer. If the offeree alters the original offer in non-material aspects, the offer is still accepted and becomes automatically effective, unless the offerer explicitly objects. Similarly, dissent subsumes the cases where an offer is ignored until it expires or where the offeree changes the original offer in material aspects. The automaton depicted in Figure 1 does not explicitly model timely behavior, such as that every offer is equipped by a validity time, whereas it includes specifically material alterations to an offer.

Material alterations are determined as contractual terms related to price, payment, quality and quantity of the goods, place and time of delivery, liability determinations, and settlement of disputes [11]. A materially altered offer is automatically rejected and the original order is replaced by the new counter-offer. In Figure 1 this is reflected by returning to the respective initial state of an offer. In contrast to a non-materially altered offer, the counter-offer does not automatically become effective, but requires explicit assent by the new offeree (the former offerer). 


\subsection{Conflicts of Laws}

Seven major legal traditions are identified and presented in [1]. These comprise the chthonic (customary), several religiously inspired and the western (civil law and common law) legal systems. They emerged from different contexts and regions, whereas they mixed partially over time or exist in parallel in some places. Their respective characteristics conflict in several aspects so that full integration is hardly achievable-be it only for specific sub-areas of commerce law. Incompatibilities prevail in traditional cases as much as they become apparent, when considering electronic service provisioning in the Internet. Due to the Internet's global reach the chance for situations, where legal traditions collide in an incompatible way, is even increased. It is the concern of the so-called conflicts of laws, also referred to as private international law, to decide on the question what jurisdiction applies in a specific case.

[1] concludes as "The answer would appear to be that there is no such universalizable core. This is good news for the sustainability of the major, complex, legal traditions of the world." Accordingly and as a matter of last resort, commercial electronic service provision might be declined at all under unresolvable conditions. The approach of roman law which separated into two branches (ius civile applicable to Romans and ius gentium applicable to non-romans) is, however, by no means a solution to conflicts of laws in the Internet. Simply ignoring such conflicts is not feasible in a global context. It would only be feasible, if there was a globally accepted, specific Internet law that could be separated from existing legal systems.

Chances for such an Internet law that is globally accepted are assessed as being low, since as it would collide with national sovereignity. On the other hand, United Nations law on international trade of goods as modeled in Figure 1 is one effort to overcome incompatibilties, whereas it reflects those principles of western commerce law most directly. This is of high relevance since territoriality is not an accepted prinicple in every legal system. Territoriality, however, is a fundamental principle for commerce law in western law systems as legal institutions are recognized in western law as state authorities [1].

Overall, the potential of unresolvable conflicts of laws is fully acknowledged. It is valued as a general open issue in the legal domain that this work needs to be fully aware of. However, it is not the task of this work to determine a solution to it in the most general way. In contrast, this work seeks for a technical solution to conclude legally compliant contracts in the Internet. This solution needs to be based on established legal principles of conflicts of laws. It, thus, aims to implement these principles technically, but it does not seek to change underlying legal principles.

\subsection{Internet Organization}

Internet design principles, in terms of protocols in use and its organizational composition, as well as dynamics in Internet routing mechanisms determine the key technical background to be investigated. The first area reflects the extra-territorial nature of the Internet, being composed from various administrative domains, but lacking any geographically bound addressing elements. The second area characterizes mechanisms applied in laying out a communications path through intermediate systems from any end system to another. 
The Internet is organized as a network of networks. Each of them determines an administrative domain with its own routing policy, whereas actual internal routing details remain undiscovered by others. Traditionally, such a domain is termed autonomous system (AS) [10], identified by a unique number (AS number, ASN). Since ASNs are scarce, it is possible that smaller domains receive a private ASN not visible to all other ASes [4].

Within an AS, the same intra-AS routing protocol (also referred to as Interior Gateway Protocol, IGP) is used whereas inter-AS protocols (Exterior Gateway Protocol, EGP, [10]) regulate routing between border routers of different ASes. The term AS evolved over time from its original perception as a set of systems that make use of the same IGP towards the wider understanding of an administrative domain [2]4]. Routing in this context refers to network routing on layer 3, but it sometimes subsumes in a wider understanding also layer 2 switching, which establishes point-to-point connectivity. When a packet needs to be sent from one end system (ES) [2] to another, routing mechanisms lay out the way the packet takes on its journey through intermediate systems (IS) [2] in terms of routers and switches.

In case a (two-party) contract for a commercial value added service shall be concluded, contractual parties are represented by one ES each. Accordingly, contractual parties are each member of an AS. This implies that contractual parties are identifiable by means of an IP address assigned to the respective ES. Accordingly, ASes are identifiable by the respective ASN a given IP address belongs to. However, the respective territory, or any dependable information on a party's (i.e. the respective ES's) geographical location cannot be determined from knowing about a party's AS. Nor is it possible to establish a clear relation between ASes and territories. Contractual parties can either belong to the same AS or to different ASes. Equally, they can reside in the same or different territories. ASes in turn can be fully contained within one territory or they can span multiple (two or more) territories. Moreover, routing mechanisms, influence legal determinations to be considered as conveyed data passes one or several territories. The latter is assumed to take place primarily in cases where contractual parties reside in distant locations, but it can also happen in case parties are associated with the same AS. Routing, thus, shows a high impact on geographical and territorial issues.

Ambiguity in relating ASes to territories constitutes the very essence of the Internet's extra-territorial nature. This design principle collides directly with a regulation's validity that is bound to a geographically limited area. Therefore, legal compliance for contracts in the Internet cannot be accomplished without a mechanism that allows for a reliable mapping of ASes to territories, rendering the Internet into a location-aware infrastructure. This allows to address the problem whether contractual parties can conclude an agreement under given circumstances.

\section{Problem Statement}

Commercial offerings of value added services in the Internet base on contractual agreements. Applicable contractual terms are expressed in Service Level Agreements (SLA) and technically refined by individual metrics (Service Level Objectives, SLO), whereas a Service Level Specification (SLS) is perceived as a technically interpreted SLA with 
its SLOs for a given contract [13]. In accordance with those arguments outlined with respect to legal compliance in an international context ( $c f$. Sections 2.1 and 2.2) and with respect to administrative and legal domains ( $c f$. Section 2.3), two problems are determined as follows:

I) For given parties (in terms of legal entities) and a given electronic service to be provided, a decision has to be taken, if at all and under which governing law a contract can be concluded.

II) Once a contract was agreed upon, the service provision itself needs to be legally compliant with the governing law in question.

Legal compliance in relation to problem \refers to commerce law requirements on concluding contracts and in particular to private international law (conflicts of laws). Legal compliance in relation to problem refers accordingly to compliance with negotiated contractual terms as characterized by SLS conditions. In order to conduct automated compliance checks on both, problems \and $\Pi$ two distinct prerequisites are identified:

1) A mapping mechanism from AS to territories is needed.

2) Guaranteed associations between contractual partners need to be taken.

Mapping from AS to territory (or potentially territories) allows contractual parties that are located in an AS (the same AS or different ASes) to be assigned to legal domains. If contractual parties are not bound to a specific location, i.e. they are mobile, such a mapping relation needs to deal with dynamics in a flexible manner. Mobility increases additionally the complexitity in attributing unambiguously contractual parties to geographical locations.

While prerequisite 1 focuses primarily on the mutual end points of an electronic service provided, prerequisite 2 considers particularly the conveyance of data in between. Due to dynamic routing, no guarantees of persistent associations can be assumed [9]. Figure 2 shows a simplified arrangement of two contractual parties A and B, both located in the same legal domain (LD), where exchanged packets can be routed along one of two possible paths. The association denoted by a dotted line passes another legal domain, where different, potentially conflicting, legal determinations exist. Therefore, ways in routing protocols and behavior need to be found that produce deterministic and reliable expectations on associations, while ensuring not to interfere with the robustness of routing mechanisms. This simplified example visualizes that legal and technical viewpoints are not easily integrated, but require a careful inspection of different scenarios covering the various configurations possible to lay out ESes and ISes, ASes and LDs, and the respective associations.

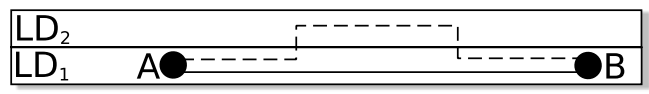

Fig. 2. Example Associations Between Contractual Partners A and B (LD =Legal Domain) 


\section{Solution Approach}

A technical solution to those problems and prerequisites outlined implies a wide range of activities in different fields to be undertaken. These activities embrace typically each the according set of subactivities, in particular exploration and analysis of existing work, design of a solution, implementation, and evaluation. Overall work status is driven by current exploration and partly design activities which will build the solid basis for upcoming implementation and evaluation phases. Detailed work status information for problems $\prod$ and $\Pi$ and for prerequisites 1 and 2 is given subsequently.

Problem 【is currently in states exploration and analysis of related work as well as design of a solution. An in-depth examination of integration options for the major legal traditions in the world was undertaken based on the comprehensive analysis in [1]. Next steps in this context include careful inspection of conflicts of laws (private international law) processes. Rules of so-called choice of law are of particular interest. Choice of law allows contractual parties to select for an agreement to be concluded what (national) law is applied to the agreement. This includes exemplarily what procedures to follow and what court to call in case of disputes. A comprehensive survey on international agreements, such as the United Nations determinations on contracts for the international sale of goods [11], started recently. This study aims at formalized rules determining under which circumstances contractual parties can use choice of law.

Based on those generalized rules determined in exploration and analysis phase, solution design with regard to problem】forsees the altered contract formation process as visualized in Figure 3 It extends the standard contract formation process ( $c f$. Figure 1) by an automated compliance check which is performed after an offer was issued. This compliance check addresses problem $\square$ as it answers whether a contract under given terms of an offer can be concluded and what law (choice of law principle) governs the agreement. Successful compliance checks transform an initial offer into a so-called lawful offer. Unsuccessful checks result in non-compliance, which imlies that a contract cannot be concluded. Whenever material alterations are made to a lawful offer or to an altered offer, resulting in rejection and a counter-offer, a new successful compliance check is required in order to render the counter-offer into lawful state.

Similar to problem $\rrbracket$ problem $\prod$ is in states exploration and analysis of related work as well as design of a solution. In order to outline the key characteristics for legal compliance in specific application fields, the domain of mobile grid applications was

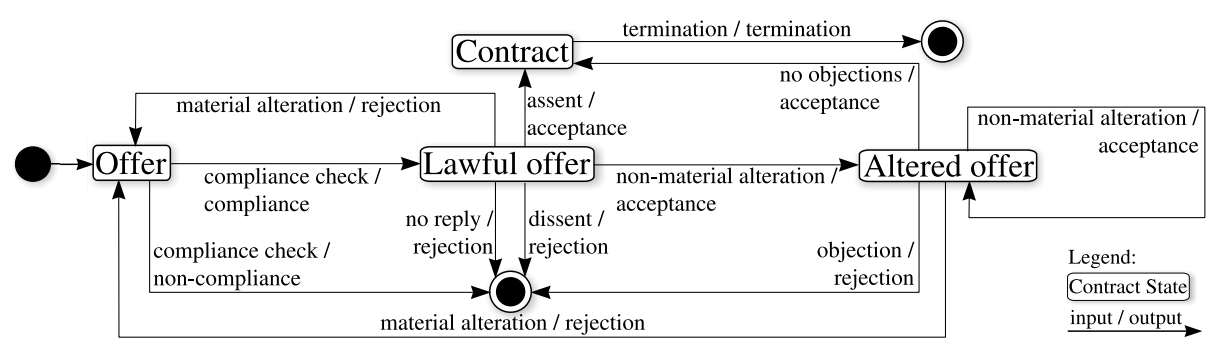

Fig. 3. Altered Contract Formation Finite State Machine 
investigated initially [12] and a comprehensive follow-up study is currently conducted. This study focuses on the relevant set of European regulations for commercial value added services, namely policies governing electronic commerce, consumer protection, copyright and related rights, cybercrime, and data protection. Solution design with regard to problem $\Pi$ applies a second type of automated compliance checks. These checks show typical characteristics of auditing tasks. Legal compliance checks are conducted by means of automated SLA compliance checks for regulations-specific metrics [3].

Prerequisites 1 and 2 are currently in exploration and analysis of related work state. For prerequisite 1, investigations on promising approaches are ongoing. This includes mechanisms to retrieve location information from the TCP/IP protocol stack (e.g., derived from latency measurements based on Traceroute [7] or by leveraging a locationverified care-of address as used in mobile IP) or from technical equipment (e.g., by means of certificates provided by a network operator). Similarly, the selection process of candidates to provide the basic mechanisms for a solution to prerequisite 2 started recently. Based on studies on route stability in the Internet [9], Multi Protocol Label Switching (MPLS, [5]) is investigated as a means for laying out a signaled association between contractual partners.

\section{References}

1. Glenn, H.P.: Legal Traditions of the World: Sustainable Diversity in Law, 2nd edn., pp. 1432. Oxford University Press, Oxford (2004)

2. Hares, S., Katz, D.: Administrative Domains and Routing Domains. A Model for Routing in the Internet RFC 1136, pp. 1-10 (December 1989)

3. Hasan, H., Stiller, B.: A Generic Model and Architecture for Automated Auditing. In: Schönwälder, J., Serrat, J. (eds.) DSOM 2005. LNCS, vol. 3775, pp. 121-129. Springer, Heidelberg (2005)

4. Hawkinson, J., Bates, T.: Guidelines for Creation, Selection, and Registration of an Autonomous System (AS). RFC 1930, pp. 1-10 (March 1996)

5. IETF MPLS working group: Multiprotocol Label Switching (MPLS) (March 2006), http: / / www . ietf .org/html. charters/mpls-charter.html

6. Karsten, M., Schmitt, J., Stiller, B., Wolf, L.: Charging for Packet-switched Network Communication - Motivation and Overview. Computer Communications 23(3), 290-302 (2000)

7. Malkin, G.: Traceroute Using an IP Option. RFC 1393, pp. 1-7 (October 1993)

8. Morariu, C., Waldburger, M., Stiller, B.: An Integrated Accounting and Charging Architecture for Mobile Grids. In: Third International Workshop on Networks for Grid Applications (GridNets 2006), San Jose (CA), USA, pp. 1-10 (October 2006)

9. Paxson, V.: End-to-end Routing Behavior in the Internet. ACM SIGCOMM Computer Communication Review 36(5), 41-56 (2006)

10. Rosen, E.C., Beranek, B.: Newman Inc: Exterior Gateway Protocol (EGP). RFC 827, pp. 1-44 (October 1982)

11. United Nations Commission on International Trade Law (UNCITRAL): United Nations Convention on Contracts for the International Sale of Goods (CISG), pp. 1-47 (April 1980)

12. Waldburger, M., Stiller, B.: Regulatory Issues for Mobile Grid Computing in the European Union. In: 17th European Regional ITS Conference, pp. 1-9 (August 2006)

13. Westerinen, A., et al.: Terminology for Policy-Based Management. RFC 3198, pp. 1-21 (November 2001) 